\title{
Circulating Microparticles in Patients with Symptomatic Carotid Disease Are Related to Embolic Plaque Activity and Recent Cerebral Ischaemia
}

\author{
Neghal Kandiyila, d Shane T. MacSweeney ${ }^{b}$ Stan Heptinstall ${ }^{c}$ Jane May ${ }^{c}$ \\ Susan C. Fox ${ }^{\mathrm{c}}$ Dorothee P. Auer ${ }^{\mathrm{a}}$ e \\ aRadiological Sciences in the Faculty of Medicine and Health Sciences, University of \\ Nottingham, Nottingham, UK; b Vascular Surgery, Nottingham University Hospitals, \\ Nottingham, UK; ${ }^{c}$ Cardiovascular Medicine, Division of Clinical Neurosciences, University of \\ Nottingham, Nottingham, UK; ${ }^{d}$ University Hospitals of Leicester NHS Trust and University \\ of Leicester, Leicester, UK; eNIHR Nottingham BRC, Nottingham, UK
}

\section{Keywords}

Platelet microparticles - Endothelial microparticles - Magnetic resonance imaging of carotid plaque haemorrhage - Brain diffusion magnetic resonance imaging - Cerebral ischaemia . Transcranial Doppler ultrasound · Micro-embolic signals · Amaurosis fugax

\begin{abstract}
Background and Purpose: In order to assess the association of microparticles derived from activated platelets (PMP) or endothelial cells (EMP) with risk markers for recurrent embolic events in patients with symptomatic carotid artery disease, we studied the associations between PMP/EMP and three risk markers: plaque haemorrhage $(\mathrm{PH})$, micro-embolic signals and cerebral diffusion abnormalities. Methods: Patients with recently symptomatic high-grade carotid artery stenosis (60-99\%, 42 patients, 31 men; mean age $75 \pm 8$ years) and 30 healthy volunteers (HV, 11 men; mean age $56 \pm 12$ years) were prospectively recruited. Patients were characterised by carotid magnetic resonance imaging (presence of PH [MRI PH]), brain diffusion MRI (cerebral ischaemia [DWI+]) and transcranial Doppler ultrasound (micro-embolic signals [MES+]). PMP and EMP were classified by flow cytometry and expressed as log-transformed counts per microlitre. Results: MES+ patients $(n=18)$ had elevated PMP (MES+ $9.61 \pm 0.57)$ compared to HV $(8.80 \pm 0.73 ; p<0.0001)$ and to MES- patients $(8.55 \pm 0.85 ; p<$ $0.0001)$. Stroke patients had elevated PMP $(9.49 \pm 0.64)$ and EMP $(6.13 \pm 1.0)$ compared to non-stroke patients (PMP $8.81 \pm 0.73, p=0.026$, EMP $5.52 \pm 0.65, p=0.011$ ) and HV (PMP $8.80 \pm 0.73, p=0.007$, and EMP $5.44 \pm 0.47, p=0.006)$. DWI+ patients $(n=16)$ showed elevated PMP (DWI+ $9.53 \pm 0.64 ;$ vs. HV, $p=0.002)$ and EMP (DWI+ $5.91 \pm 0.99$ vs. HV $5.44 \pm 0.47 ;$
\end{abstract}


$p=0.037)$. Only PMP but not EMP were higher in DWI+ versus DWI- patients $(8.67 \pm 0.90$; $p=0.002$ ). No association was found between PMP and EMP with MRI PH. Conclusions: PMP and EMP were associated with stroke and recent cerebrovascular events ( $\mathrm{DWI}+$ ) but only PMP were also associated with ongoing (MES+) thrombo-embolic activity suggesting a differential biomarker potential for EMP to index cerebral ischaemia while PMP may predict on-going thrombo-embolic activity.

(C) 2019 The Author(s)

Published by S. Karger AG, Basel

\section{Introduction}

Antiplatelet medication and carotid endarterectomy are effective for secondary stroke prevention $[1,2]$; however, there are subgroups of patients who fail to respond to medical treatment $[3,4]$. This well-recognised heterogeneity in treatment responsiveness may be linked to individual differences in platelet function, but to date there is no established platelet function test that could be recommended for clinical use [3]. Ideally, a platelet function test would allow to predict the individual's risk of stroke and likelihood to respond to specific antiplatelet drugs, and it would allow to directly monitor the treatment response.

There are several biomarkers with predictive power of the risk of stroke in patients with carotid artery disease, such as magnetic resonance imaging (MRI) of carotid plaque haemorrhage (PH) $[4,5]$. Whilst MRI PH can be considered a marker of the risk of thromboembolic activity [6], it does not inform on the mechanism of thrombus formation itself, and we do not know its interrelation with platelet-derived microparticles (PMP). Other imaging markers such as diffusion-weighted imaging (DWI) of the brain has a high sensitivity (90-100\%) and specificity (97-100\%) for detecting cerebral ischaemia in clinical stroke [7-9] and approximately 30\% in transient ischaemic attack (TIA) [10-12]. Importantly, demonstration of an index ischaemic event via DWI has been shown to predict recurrent stroke $[10,11]$. Another widely accepted risk marker is micro-embolic signals (MES) directly reflecting on-going cerebral thrombo-embolic activity when using transcranial Doppler ultrasound (TCD) monitoring $[13,14]$.

Additional mechanistic biomarkers would be desirable to inform on the stroke risk amenable to specific antiplatelet therapy. Microparticles (MP) are promising in this context and have been proposed as biomarker of thrombo-embolic risk [15]. PMP and endothelial MP (EMP) are membranous phospholipid vesicles (size $0.05-2 \mu \mathrm{m}$ ) that are derived from platelets and red blood cells. MP contain an antigen complex and therefore can be quantified, separated from each other and from inflammatory cells. They are complex molecules with complex interactions that have procoagulant, pro-inflammatory and pro-atherogenic properties [16, 17] (see online suppl. Fig. 1; see www.karger.com/doi/10.1159/000495942 for all online suppl. material). These characteristics are essential in the pathophysiology of thromboembolic stroke. There is now increasing evidence that PMP [18-21] are also elevated in ischaemic stroke and EMP in unstable plaques [22].

MP are therefore attractive biomarker candidates to probe the responsiveness or resistance to conventional antiplatelet treatment and moreover to directly assess the mechanistic efficacy of novel drugs targeting platelet function.

In this study, we aimed to investigate the potential biomarker role of PMP and EMP in patients with recent symptomatic carotid artery stenosis. Specifically, we assessed whether and which type of MP may be associated with (i) recent cerebrovascular ischemic events, indexed by diffusion MRI, (ii) ongoing thrombo-embolic activity (MES) and (iii) long-term thrombo-embolic risk indexed by MRI PH. 


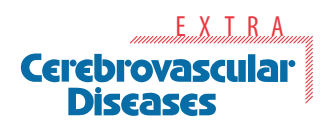

\begin{tabular}{l|l}
\hline \multicolumn{2}{l}{ Cerebrovasc Dis Extra 2019;9:9-18 } \\
\hline DOI: $10.1159 / 000495942$ & $\begin{array}{l}\text { @ 2019 The Author(s). Published by S. Karger AG, Basel } \\
\text { www.karger.com/cee }\end{array}$ \\
\hline
\end{tabular}

Kandiyil et al.: Microparticles and Embolic Plaque Activity

\section{Methods}

\section{Patient and Healthy Volunteer Recruitment}

All patients gave informed written consent and were prospectively recruited from a TIA/ stroke clinic. The research protocol was approved by the Nottingham ethics committee 2 .

Forty-four patients with symptomatic carotid stenosis $(\geq 60 \%)$ [23] were prospectively recruited from a TIA or stroke clinic, where the TIA, amaurosis fugax (AmF) or stroke was confirmed by a stroke physician or a neurologist with a specialised interest in stroke. Patients were excluded if they had less than $60 \%$ carotid stenosis, asymptomatic carotid disease/ischaemic event more than 6 months from time of recruitment, known haematological disorder, if the MRI would delay surgery, had treatment with a thrombolytic prior to recruitment, an ongoing inflammatory or infective medical condition or any contraindication to MRI.

A control group of healthy volunteers (HV) was also recruited. They were subdivided into older ( $>55$ years) (OHV) and younger healthy volunteers (YHV) to assess any age influence on MP. A careful medical history of vascular and demographic risk factors for both groups was recorded. Inclusion criteria: (1) no significant carotid stenosis $(<30 \%)$ confirmed by duplex ultrasound (if volunteer was over 50 years). Exclusion criteria: (1) history of cardiac or peripheral vascular disease, (2) history of TIA or stroke, (3) known haematological disorder, (4) ongoing inflammatory or infective medical condition.

\section{TCD Protocol}

Patients had non-mobile TCD to detect MES (mean duration of scan: $49 \mathrm{~min}$ ) which was performed separately but on the same day as the MRI. This was achieved using new-generation transcranial M-mode imaging (Doppler box, DWL Elektronische Systeme GmbH, Germany).

\section{MRI Protocol}

Imaging was performed at $1.5 \mathrm{~T}$ (Signa, GE Healthcare, WI, USA) using standard receiveonly quadrature neck array coils. As published previously [24], a coronal T1-weighted 3-dimensional gradient echo sequence with effective blood nulling and fat suppression was used to identify areas of complicated plaque, specifically PH. DWI MRI was performed with an 8-channel neurovascular coil [6] by using an axial fat-suppressed single-shot double-spinecho echo-planar imaging sequence with 15 diffusion gradient directions and high spatial resolution $(1.5 \times 2 \times 3)$.

\section{Blood Protocol for Analysis of MP}

Blood was taken on the same day prior to MRI/TCD from the antecubital vein via nonvacuum syringes using a 21-G needle and placed in 5-mL citrate anticoagulant bottles. The blood samples were analysed in Cardiovascular Medicine at the University of Nottingham, by or under direct supervision of experienced researchers (S.C.F. or J.M.) who were blinded to TCD and MRI findings. Within $10 \mathrm{~min}$ of collection, the samples underwent a standard double centrifugation process. Platelet-rich plasma was prepared by centrifugation for $6 \mathrm{~min}$ at $1,000 \mathrm{~g}$. The platelet-rich plasma was removed with a Pasteur pipette, and the remaining platelet-poor plasma (PPP) was centrifuged at $180 \mathrm{~g}$ for $10 \mathrm{~min}$ at room temperature. The PPP supernatant was then transferred to microtubes and was frozen at $-80^{\circ} \mathrm{C}$ and thawed for 30 min prior to analysis. A preliminary study performed in the department showed no elevation of PMP over time and no significant degradation once frozen, from day 1 compared to day 21 in HV ( $n=3$, PMP: CV 5\%, EMP: CV 9\%). 

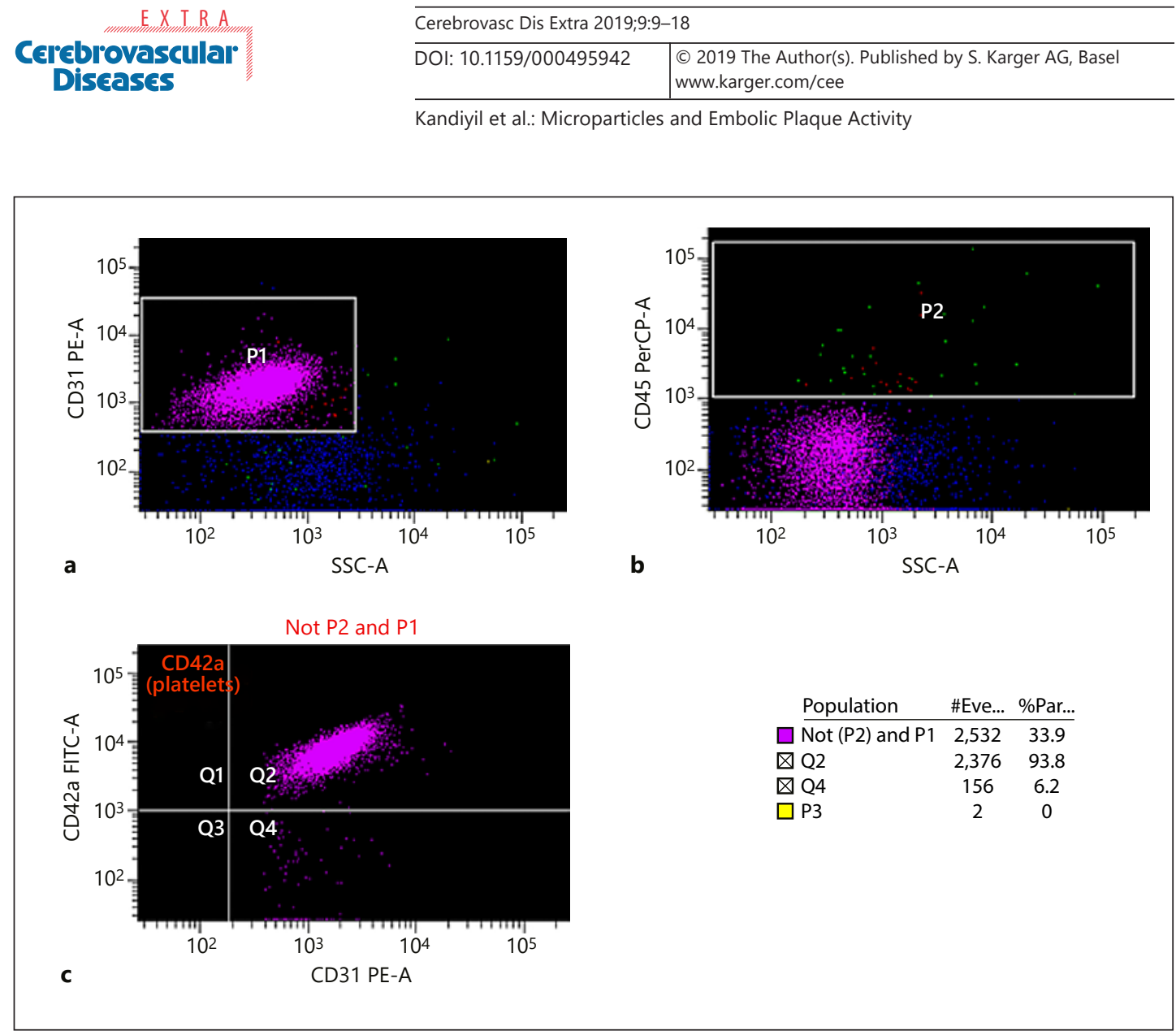

Fig. 1. The detection of platelet-derived/endothelial microparticles (PMP/EMP) by flow cytometry. Counts calculated as PMP and EMP per microlitre using flow cytometry showing population data. \#Eve, number of events; \%Par, percent particles. a CD31 fluorescent conjugated antibodies were used to detect all MP (P1). b CD45 fluorescent conjugated antibodies were used to exclude lymphocytes (P2). c CD42a fluorescent conjugated antibodies were used to detect PMP which were excluded from P1 and P2, and the remaining MP were measured as EMP.

By using cell-specific antibodies, simultaneous detection of the different types of MP was possible. CD31 antibody was used for detection of both PMP and EMP; CD42a selected only PMP, and CD45 was used for exclusion of any remaining leucocytes. At room temperature the PPP was incubated with antibodies CD31, CD45, CD42a $(3 \times 10 \mu \mathrm{L})$ for $25 \mathrm{~min}$ at $4{ }^{\circ} \mathrm{C}$ in the dark. $500 \mu \mathrm{L}$ of FACS flow (flow cytometry buffer) was added to the PPP samples, which were then counted for exactly $30 \mathrm{~s}$ by flow cytometry (BD LSR II; Fig. 1). This analysis was performed in triplicate and MP counts calculated as counts per microlitre using Trucount standard beads.

\section{Statistical Methods}

PMP and EMP were expressed as counts per microlitre, and the median PMP/EMP counts from the triplicated results were used. Since PMP/EMP were not normally distributed (according to Shapiro-Wilk test $<0.001$ ) the results were log transformed (log median PMP/ EMP counts). Groups were compared using independent $t$ tests and ANCOVA as appropriate.

Potential demographic influences (age and sex) on MP were explored in the HV group by respective subgroup comparison of PMP/EMP and age vs. EMP/PMP scatter plot. As a further caveat against bias from age and sex, repeat tests controlling for age and sex were carried out in patient comparisons to all HV using univariate analysis of covariance (ANCOVA) with multiple test corrections using the Bonferroni test. 
Table 1. Platelet (PMP) and endothelial (EMP) derived microparticles are associated with acute embolic cerebral ischaemia

\begin{tabular}{llllll}
\hline & $\begin{array}{l}\text { DWI+ } \\
(n=16)\end{array}$ & $\begin{array}{l}\text { DWI- } \\
(n=26)\end{array}$ & $\begin{array}{l}\text { HV } \\
(n=30)\end{array}$ & $\begin{array}{l}\text { DWI+ vs. HV } \\
(p \text { value })\end{array}$ & $\begin{array}{l}\text { DWI+ vs. DWI- } \\
(p \text { value })\end{array}$ \\
\hline PMP & $9.53 \pm 0.64$ & $8.67 \pm 0.90$ & $8.80 \pm 0.73$ & $0.002(F=11.0)$ & $0.002(F=10.7)$ \\
\hline EMP & $5.91 \pm 0.99$ & $5.65 \pm 0.83$ & $5.44 \pm 0.47$ & $0.037(F=4.7)$ & 0.32 \\
\hline
\end{tabular}

Microparticles per microlitre log-transformed, means with standard deviation. DWI, diffusion-weighted imaging; HV, healthy volunteers. Italics indicate significant $p$ values with ANCOVA $(F)$.

Table 2. Platelet (PMP) and endothelial (EMP) derived microparticles are associated with stroke

\begin{tabular}{llllll}
\hline & $\begin{array}{l}\text { Stroke } \\
(n=12)\end{array}$ & $\begin{array}{l}\text { Non-stroke } \\
(n=30)\end{array}$ & $\begin{array}{l}\text { HV } \\
(n=30)\end{array}$ & $\begin{array}{l}\text { Stroke vs. HV } \\
(p \text { value })\end{array}$ & $\begin{array}{l}\text { Stroke vs. non-stroke } \\
(p \text { value })\end{array}$ \\
\hline PMP & $9.49 \pm 0.64$ & $8.81 \pm 0.73$ & $8.80 \pm 0.73$ & $0.007(F=8.1)$ & $0.026(F=5.3)$ \\
EMP & $6.13 \pm 1.0$ & $5.52 \pm 0.65$ & $5.44 \pm 0.47$ & $0.006(F=8.3)$ & $0.011(F=6.8)$ \\
\hline
\end{tabular}

Microparticles per microlitre log-transformed, means with standard deviation. HV, healthy volunteers. Italics indicate significant $p$ values with ANCOVA $(F)$.

Table 3. Platelet-derived (PMP) but not endothelial (EMP) microparticles are associated with multiple embolic signals (MES)

\begin{tabular}{llllll}
\hline & $\begin{array}{l}\text { MES }+ \\
(n=18)\end{array}$ & $\begin{array}{l}\text { MES- } \\
(n=24)\end{array}$ & $\begin{array}{l}\text { HV } \\
(n=30)\end{array}$ & $\begin{array}{l}\text { MES+ vs. HV } \\
(p \text { value })\end{array}$ & $\begin{array}{l}\text { MES+ vs. MES- } \\
(p \text { value })\end{array}$ \\
\hline PMP & $9.61 \pm 0.57$ & $8.55 \pm 0.85$ & $8.80 \pm 0.73$ & $<0.0001(F=19.5)$ & $<0.0001(F=16.3)$ \\
EMP & $5.70 \pm 0.76$ & $5.78 \pm 0.99$ & $5.44 \pm 0.47$ & 0.16 & 0.65 \\
\hline
\end{tabular}

Microparticles per microlitre log-transformed, means with standard deviation. HV, healthy volunteers. Italics indicate significant $p$ values with ANCOVA $(F)$.

MES and DWI change with time from the initial ischaemic event [25-27], that was therefore controlled when comparing PMP and EMP levels in MES, DWI and MRI PH subgroups. $p<0.05$ was regarded as statistically significant. Statistical calculations were made using SPSS software (SPSS 20.0; SPSS, Chicago, IL, USA).

\section{Results}

\section{General Findings}

Forty-four patients were recruited, of whom 2 were excluded due to claustrophobia. Forty-two patients ( 31 men; mean age $75 \pm 8$ years, range 56-88) and $30 \mathrm{HV}$ (11 men; mean age $56 \pm 12$ years, range $23-72$ ) were included.

Demographics and vascular risk factors are shown in online supplementary Table 1. 
Kandiyil et al.: Microparticles and Embolic Plaque Activity

There were no significant differences for EMP or PMP between the two age groups: PMP (OHV: $8.74 \pm 0.8$ and YHV: $8.90 \pm 0.62$; non-significant [ns]) or EMP (OHV: $5.35 \pm 0.43$ and YHV: $5.61 \pm 0.52 ; \mathrm{ns}$ ). There was no significant sex influence on PMP (men $9.05 \pm 0.78$ vs. women $8.65 \pm 0.68$; ns) or EMP (men $5.41 \pm 0.45$ vs. women $5.48 \pm 0.49$; ns).

\section{PMP and EMP Are Associated with Acute Embolic Cerebral Ischaemia}

There were no differences in PMP or EMP between the patients and HV. Subtype analysis revealed that patients with acute cerebral ischaemia (DWI+) lesions $(n=16)$ had elevated PMP and EMP compared to HV ( $p=0.002$ and $p=0.037$, Table 1 ) but only PMP was significantly elevated in patients with acute cerebral ischaemia (DWI+) compared to no DWI lesions (DWI-, $n=26, p=0.002$, Table 1). Also, patients presenting with stroke $(n=12)$ had elevated PMP and EMP compared to HV ( $p=0.007$ and $p=0.006)$ and compared to non-stroke patients (Table 2). The difference between DWI+ and DWI- patients remained significant for PMP ( $p=0.035)$, when additionally controlling for stroke. There was no correlation for DWI+ patients between time from ischaemic event to blood sampling of PMP $(r=0.32, p=0.23)$ and EMP $(r=0.33, p=0.22)$.

PMP Are Associated with On-Going Embolic Activity, but Not Underlying Long-Term Thrombo-Embolic Risk (MRI PH)

MES + patients $(n=18)$ had significant elevation in PMP compared to MES- patients $(n=$ 24) $(p<0.0001)$ and HV $(p<0.0001$, Table 3$)$. EMP were not altered by MES status (ns, Table $3)$. The difference remained significant $(F=16 ; p<0.0001)$ when also controlling for stroke. Carotid PH was not associated with changes in PMP and EMP (ns, online suppl. Table 2).

\section{Vascular Risk Factors and MPS}

From the studied vascular risk factors, only ischaemic heart disease was associated with significantly elevated EMP ( $p=0.002$, online suppl. Table 1$)$.

\section{Antiplatelet Treatment and MP}

There were no significant differences in PMP between patients with and without antiplatelet medication. There was a tendency towards higher PMP counts in patients without antiplatelet medication compared to $\operatorname{HV}(p=0.08)$.

EMP were significantly elevated in patients without antiplatelet medication compared to those who were on antiplatelet medication $(p=0.03)$ and compared to $\mathrm{HV}(p=0.004)$.

\section{Discussion}

PMP were higher in patients with recent thrombo-embolic activity indexed as DWI+, ongoing micro-embolisation (MES+) or when presenting with stroke. EMP were only elevated in DWI+ and stroke patients.

In patients with recently symptomatic carotid stenosis of at least $60 \%$ we did not observe alterations of MP for the whole patient group. This finding may at first be surprising but is in line with several other studies $[28,29]$ that have shown no significant differences in PMP in patients compared to HV. There is an apparent conflict with Kuriyama et al. [19] and Cherian et al. [21], who reported higher PMP levels in their patient groups. However, all of their patients presented with stroke, which suggests that higher PMP levels may lead to a greater severity of cerebral ischaemia burden and explain our observed differences. In fact, most of our patients did not present with stroke, and those who did also demonstrated high PMP.

Further support for a link between recent cerebral ischaemia and PMP comes from our observation that patients with imaging-confirmed acute or subacute cerebral ischaemia as 
indexed by DWI+ lesions had significantly elevated PMP compared to HV and to those without imaging-confirmed ischaemia. DWI+ lesions in patients presenting with either AmF/TIA or stroke symptoms are arguably a clearer definition of recent cerebral ischaemia than duration of symptoms defining stroke [7]. In contrast, DWI- patients may include patients with nonischaemic pathology (stroke/TIA mimic) [7] or reflect a mild ischaemic burden [30] or too long an interval from the presenting symptom. DWI+ patients are increasingly considered to have a similar risk as stroke patients [31]. We hence also looked at MP levels in the subgroup of stroke patients and confirmed elevated MP in stroke patients compared to those presenting with TIA or AmF alone. Taken together our study confirms and extends previous reports on elevated PMP in acute stroke patients $[19,21]$ demonstrating MP elevation in recent cerebral ischaemia only when evidenced by DWI+ or lasting longer than $24 \mathrm{~h}$.

We found a strong association between PMP and micro-embolic signals indexing ongoing thrombo-embolic activity. This finding supports a direct pathophysiological link between PMP and cerebral emboli. There is experimental evidence that PMP are generated by platelet activation or apoptosis induced by various stimuli such as atherosclerosis [32], shear stress [33] and vascular risk factors such as smoking [34]. This is combined with a PMP potent thrombogenic effect around its membrane wall along with receptors that are important factors in initiation of the coagulation cascade [35]. It suggests PMP can exert its procoagulant effects distant to the site of platelet activation/apoptosis, for longer periods than platelet activation.

PMP/EMP were not significantly elevated in MRI PH+ patients. The lack of association with vulnerable plaque features such as PH make PMP/EMP unlikely as causative factors of $\mathrm{PH}$ or as mediators of the $\mathrm{PH}$-associated elevated thrombo-embolic risk. This discrepancy may be explained by the divergent dynamic behaviour of MRI PH which remains relatively stable for at least 1 year [36] whilst changes of PMP elevation beyond 3 months are less clear $[18,21]$.

We found that stroke patients showed higher EMP compared to healthy controls. This is well in line with two previous studies reporting elevated levels of EMP in stroke patients compared with HV $[28,29]$. We also found higher EMP in stroke versus non-stroke patients. EMP were also higher in DWI+ patients versus controls but not compared to DWI- patients. This is in line with reported similar levels of EMP between patients with stroke and stroke mimic (DWI-) patients [37]. The differences between PMP and EMP are likely to be related to the differences in MP origin, as EMP arise from endothelial cells and have different physiological characteristics compared to PMP. The lack of association between EMP and MES in contrast to a strong association between PMP and MES points to further pathophysiological differences with EMP being largely unrelated to on-going micro-thrombo-embolic activity. We can however not exclude an association with certain other EMP subtypes [28] not assessed in this study.

We observed higher levels of EMP in patients without antiplatelet medication compared to those on antiplatelet medication. This putative treatment effect could further explain why EMP were not significantly elevated in our whole patient group compared to controls as most patients were treated. Interestingly, EMP increase in patients with stroke, or DWI+ lesions seemed unrelated to antiplatelet medication. The sample size was too small to further evaluate heterogeneity of medication effects, but our preliminary finding warrants further investigation of EMP as a putative marker of resistance to standard antiplatelet therapy.

In contrast, PMP levels did not differ with antiplatelet medication. This could be due to the small sample size of non-medicated patients although other studies that have also shown no significant reduction in PMP with antiplatelet medication such as aspirin [38, 39], cilostazol [38], tirofiban and eptifibatide [40]. This suggests that MP may have procoagulant 
properties that are independent of platelet function. This may be important for future studies into the mechanisms of resistance to standard antiplatelet therapy and drug development that may target MP-related coagulation pathways.

\section{Limitations of the Study}

PMP/EMP blood samples and imaging were not acquired immediately or at a constant time interval after the first cerebral ischaemic event, which will have reduced the sensitivity of TCD and DWI. Although we controlled for time from symptom to scan, we cannot exclude an effect from this in our study. We acknowledge that the median delay of 35 days may be responsible for the lack of observed elevation of PMP/EMP in patients without stroke or DWI+ lesions.

We were unable to age match our healthy controls with our patient population, but we do not believe this is likely to have affected our results, as we did not find any age or sex effect on MP in HV or patients. Moreover, reported results were confirmed by repeated tests controlling for age and sex.

\section{Conclusion}

PMP and EMP were increased in recently symptomatic carotid disease with neuroimaging-confirmed (DWI+) cerebral ischaemia or clinical stroke, while only PMP were strongly associated with ongoing (MES+) thrombo-embolic activity, and only EMP were lower in patients under antiplatelet therapy.

This study points to a differential biomarker potential of MP, with PMP to predict recent and on-going thrombo-embolic activity and EMP indicative of a recent cerebrovascular event and possibly also of insufficient antiplatelet therapy which could be tested in larger prevention treatment trials.

\section{Acknowledgements}

This research was funded and supported by the Nottingham Hospitals Charity, Nottingham Vascular Research Unit and the National Institute for Health Research Nottingham Biomedical Research Centre.

\section{Statement of Ethics}

This study had full prior approval from the ethics review committee (Nottingham Research Ethics Committee 2-06/Q2403/137).

\section{Disclosure Statement}

There are no conflicts of interest.

\section{References}

1 Collaboration AT; Antithrombotic Trialists' Collaboration. Collaborative meta-analysis of randomised trials of antiplatelet therapy for prevention of death, myocardial infarction, and stroke in high risk patients. BMJ. 2002 Jan;324(7329):71-86.

2 Rerkasem K, Rothwell PM. Carotid endarterectomy for symptomatic carotid stenosis. Cochrane Database Syst Rev. 2011 Apr;(4):CD001081.

3 Gorelick PB, Farooq MU. Advances in our understanding of "resistance" to antiplatelet agents for prevention of ischemic stroke. Stroke Res Treat. 2013;2013:727842. 
Kandiyil et al.: Microparticles and Embolic Plaque Activity

4 Hosseini AA, Simpson RJ, Altaf N, Bath PM, MacSweeney ST, Auer DP. Magnetic resonance imaging plaque hemorrhage for risk stratification in carotid artery disease with moderate risk under current medical therapy. Stroke. 2017 Mar;48(3):678-85.

5 Hosseini AA, Kandiyil N, Macsweeney ST, Altaf N, Auer DP. Carotid plaque hemorrhage on magnetic resonance imaging strongly predicts recurrent ischemia and stroke. Ann Neurol. 2013 Jun;73(6):774-84.

6 Altaf N, Goode SD, Beech A, Gladman JR, Morgan PS, MacSweeney ST, et al. Plaque hemorrhage is a marker of thromboembolic activity in patients with symptomatic carotid disease. Radiology. 2011 Feb;258(2):538-45.

7 Brunser AM, Hoppe A, Illanes S, Díaz V, Muñoz P, Cárcamo D, et al. Accuracy of diffusion-weighted imaging in the diagnosis of stroke in patients with suspected cerebral infarct. Stroke. 2013 Apr;44(4):1169-71.

8 González RG, Schaefer PW, Buonanno FS, Schwamm LH, Budzik RF, Rordorf G, et al. Diffusion-weighted MR imaging: diagnostic accuracy in patients imaged within 6 hours of stroke symptom onset. Radiology. 1999 Jan; 210(1):155-62.

9 Singer MB, Chong J, Lu D, Schonewille WJ, Tuhrim S, Atlas SW. Diffusion-weighted MRI in acute subcortical infarction. Stroke. 1998 Jan;29(1):133-6.

10 Coutts SB, Simon JE, Eliasziw M, Sohn CH, Hill MD, Barber PA, et al. Triaging transient ischemic attack and minor stroke patients using acute magnetic resonance imaging. Ann Neurol. 2005 Jun;57(6):848-54.

11 Purroy F, Montaner J, Rovira A, Delgado P, Quintana M, Alvarez-Sabín J. Higher risk of further vascular events among transient ischemic attack patients with diffusion-weighted imaging acute ischemic lesions. Stroke. 2004 Oct;35(10):2313-9.

12 Calvet D, Touzé E, Oppenheim C, Turc G, Meder JF, Mas JL. DWI lesions and TIA etiology improve the prediction of stroke after TIA. Stroke. 2009 Jan;40(1):187-92.

13 Russell D, Madden KP, Clark WM, Sandset PM, Zivin JA. Detection of arterial emboli using Doppler ultrasound in rabbits. Stroke. $1991 \mathrm{Feb} ; 22(2): 253-8$.

14 Markus HS, Brown MM. Differentiation between different pathological cerebral embolic materials using transcranial Doppler in an in vitro model. Stroke. 1993 Jan;24(1):1-5.

15 Baron M, Boulanger CM, Staels B, Tailleux A. Cell-derived microparticles in atherosclerosis: biomarkers and targets for pharmacological modulation? J Cell Mol Med. 2012 Jul;16(7):1365-76.

16 Nomura S. Function and clinical significance of platelet-derived microparticles. Int J Hematol. 2001 Dec; 74(4): 397-404.

17 Nomura S, Tandon NN, Nakamura T, Cone J, Fukuhara S, Kambayashi J. High-shear-stress-induced activation of platelets and microparticles enhances expression of cell adhesion molecules in THP-1 and endothelial cells. Atherosclerosis. 2001 Oct;158(2):277-87.

18 Lukasik M, Rozalski M, Luzak B, Michalak M, Ambrosius W, Watala C, et al. Enhanced platelet-derived microparticle formation is associated with carotid atherosclerosis in convalescent stroke patients. Platelets. 2013; 24(1):63-70.

19 Kuriyama N, Nagakane Y, Hosomi A, Ohara T, Kasai T, Harada S, et al. Evaluation of factors associated with elevated levels of platelet-derived microparticles in the acute phase of cerebral infarction. Clin Appl Thromb Hemost. 2010 Feb;16(1):26-32.

20 Lukasik M, Rozalski M, Luzak B, Michalak S, Kozubski W, Watala C. Platelet activation and reactivity in the convalescent phase of ischaemic stroke. Thromb Haemost. 2010 Mar;103(3):644-50.

21 Cherian P, Hankey GJ, Eikelboom JW, Thom J, Baker RI, McQuillan A, et al. Endothelial and platelet activation in acute ischemic stroke and its etiological subtypes. Stroke. 2003 Sep;34(9):2132-7.

22 Schiro A, Wilkinson FL, Weston R, Smyth JV, Serracino-Inglott F, Alexander MY. Elevated levels of endothelialderived microparticles, and serum CXCL9 and SCGF- $\beta$ are associated with unstable asymptomatic carotid plaques. Sci Rep. 2015 Nov;5(1):16658.

23 Sidhu PS, Allan PL. Ultrasound assessment of internal carotid artery stenosis. Clin Radiol.1997 Sep;52(9):654-8.

24 Kandiyil N, Altaf N, Hosseini AA, MacSweeney ST, Auer DP. Lower prevalence of carotid plaque hemorrhage in women, and its mediator effect on sex differences in recurrentcerebrovascular events. PLoS One.2012;7(10):e47319.

25 Srivastava AK, Mehrotra G, Bhargava SK, Agarwal S, Tripathi RP. Studies on the time course of apparent diffusion coefficient and signal intensities on T2- and diffusion-weighted MR Imaging in acute cerebral ischemic stroke. J Med Phys. 2008 Oct;33(4):162-70.

26 Lansberg MG, Thijs VN, O’Brien MW, Ali JO, de Crespigny AJ, Tong DC, et al. Evolution of apparent diffusion coefficient, diffusion-weighted, and T2-weighted signal intensity of acute stroke. AJNR Am J Neuroradiol. 2001 Apr;22(4):637-44.

27 Droste DW, Dittrich R, Kemény V, Schulte-Altedorneburg G, Ringelstein EB. Prevalence and frequency of microembolic signals in 105 patients with extracranial carotid artery occlusive disease. J Neurol Neurosurg Psychiatry. 1999 Oct;67(4):525-8.

28 Simak J, Gelderman MP, Yu H, Wright V, Baird AE. Circulating endothelial microparticles in acute ischemic stroke: a link to severity, lesion volume and outcome. J Thromb Haemost. 2006 Jun;4(6):1296-302.

29 Jung KH, Chu K, Lee ST, Park HK, Bahn JJ, Kim DH, et al. Circulating endothelial microparticles as a marker of cerebrovascular disease. Ann Neurol. 2009 Aug;66(2):191-9.

30 Hotter BA, Lechner JM, Nolte CH, Audebert HJ, Malzahn U, Heuschmann PU, et al. ABCD(2) as a screening tool for cerebral infarction on stroke MRI? Eur Neurol. 2012;67(5):315-20.

31 Giles MF, Albers GW, Amarenco P, Arsava EM, Asimos AW, Ay H, et al. Early stroke risk and ABCD2 score performance in tissue- vs time-defined TIA: a multicenter study. Neurology. 2011 Sep;77(13):1222-8. 
32 Nomura S, Nakamura T, Cone J, Tandon NN, Kambayashi J. Cytometric analysis of high shear-induced platelet microparticles and effect of cytokines on microparticle generation. Cytometry. 2000 Jul;40(3):173-81.

33 Holme PA, Orvim U, Hamers MJ, Solum NO, Brosstad FR, Barstad RM, et al. Shear-induced platelet activation and platelet microparticle formation at blood flow conditions as in arteries with a severe stenosis. Arterioscler Thromb Vasc Biol. 1997 Apr;17(4):646-53.

34 Li M, Yu D, Williams KJ, Liu ML. Tobacco smoke induces the generation of procoagulant microvesicles from human monocytes/macrophages. Arterioscler Thromb Vasc Biol. 2010 Sep;30(9):1818-24.

35 Morel O, Toti F, Hugel B, Bakouboula B, Camoin-Jau L, Dignat-George F, et al. Procoagulant microparticles: disrupting the vascular homeostasis equation? Arterioscler Thromb Vasc Biol. 2006 Dec;26(12):2594-604.

36 Kwee RM, Truijman MT, van Oostenbrugge RJ, Mess WH, Prins MH, Franke CL, et al. Longitudinal MRI study on the natural history of carotid artery plaques in symptomatic patients. PLoS One. 2012;7(7):e42472.

37 Williams JB, Jauch EC, Lindsell CJ, Campos B. Endothelial microparticle levels are similar in acute ischemic stroke and stroke mimics due to activation and not apoptosis/necrosis. Acad Emerg Med. 2007 Aug;14(8):685-90.

38 Shirafuji T, Hamaguchi H, Kanda F. Measurement of platelet-derived microparticle levels in the chronic phase of cerebral infarction using an enzyme-linked immunosorbent assay. Kobe J Med Sci. 2008 May;54(1):E55-61.

39 Choudhury A, Chung I, Blann AD, Lip GY. Elevated platelet microparticle levels in nonvalvular atrial fibrillation: relationship to p-selectin and antithrombotic therapy. Chest. 2007 Mar;131(3):809-15.

40 Goto S, Tamura N, Li M, Handa M, Ikeda Y, Handa S, et al. Different effects of various anti-GPIIb-IIIa agents on shearinduced platelet activation and expression of procoagulant activity. J Thromb Haemost. 2003 Sep;1(9):2022-30. 